\title{
To Blink or Not to Blink: Fine Cognitive Tuning of the Defensive Peripersonal Space
}

\author{
C. F. Sambo, ${ }^{1,2}$ B. Forster, ${ }^{2}$ S. C. Williams, ${ }^{3}$ and G. D. Iannetti ${ }^{1}$ \\ ${ }^{1}$ Department of Neuroscience, Physiology, and Pharmacology, University College London, London, WC1E 6BT, United Kingdom, ${ }^{2}$ Department of \\ Psychology, City University London, London, EC1V 0HB, United Kingdom, and ${ }^{3}$ Centre for Neuroimaging Sciences, Institute of Psychiatry, King's College \\ London, London, SE5 8AF, United Kingdom
}

The blink reflex elicited by the electrical stimulation of the median nerve at the wrist [hand blink reflex (HBR)] is a subcortical, defensive response that is enhanced when the stimulated hand is inside the peripersonal space of the face. Such enhancement results from a tonic, top-down modulation of the excitability of the brainstem interneurons mediating the HBR. Here we aim to (1) characterize the somatotopical specificity of this top-down modulation and investigate its dependence on (2) cognitive expectations and (3) the presence of objects protecting the face, in healthy humans. Experiment 1 showed that the somatotopical specificity of the HBR enhancement is partially homosegmental, i.e., it is greater for the HBR elicited by the stimulation of the hand near the face compared with the other hand, always kept far from the face. Experiment 2 showed that the HBR is enhanced only when participants expect to receive stimuli on the hand close to the face and is thus strongly dependent on cognitive expectations. Experiment 3 showed that the HBR enhancement by hand-face proximity is suppressed when a thin wooden screen is placed between the participants' face and their hand. Thus, the screen reduces the extension of the defensive peripersonal space, so that the hand is never inside the peripersonal space of the face, even in the "near" condition. Together, these findings indicate a fine somatotopical and cognitive tuning of the excitability of brainstem circuits subserving the HBR, whose strength is adjusted depending on the context in a purposeful manner.

\section{Introduction}

Defensive reflexes are primitive and involuntary motor responses to potentially dangerous stimuli, mediated by fast, subcortical pathways. For example, the blink reflex (BR) is consistently elicited by the electrical stimulation of the trigeminal territory but also, although less frequently, by the intense electrical stimulation of the median nerve [hand blink reflex (HBR)] (Alvarez-Blanco et al., 2009; Sambo et al., 2012). The HBR has an onset latency of $\sim 45 \mathrm{~ms}$ and, similarly to the late R2 component of the trigeminofacial BR, is entirely mediated by subcortical circuits at brainstem level (Miwa et al., 1996; Valls-Solé et al., 1997; León et al., 2011).

We demonstrated that the HBR is modulated by the proximity of the stimulated hand to the face, being dramatically increased when the hand is inside the peripersonal space surrounding the face (Sambo et al., 2012). This effect results from a tonic, topdown modulation of the brainstem circuits mediating the HBR. We proposed that such modulation is exerted by associative cortical areas (such as the premotor cortex and the ventral intraparietal area) involved in representing the peripersonal space and mapping the location of somatosensory stimuli into an external

Received Feb. 8, 2012; revised July 3, 2012; accepted July 7, 2012.

Author contributions: C.F.S. and G.D.I. designed research; C.F.S. performed research; C.F.S. and G.D.I. analyzed data; C.F.S., B.F., S.C.W., and G.D.I. wrote the paper.

The authors declare no competing financial interests.

Correspondence should be addressed to Dr. C. F. Sambo and Dr. G. D. lannetti, Department of Neuroscience, Physiology, and Pharmacology, University College London, Medical Sciences Building, Gower Street, London WC1E 6BT,UK.E-mail: c.sambo@ucl.ac.uk; g.iannetti@ucl.ac.uk.

DOI:10.1523/JNEUROSCI.0607-12.2012

Copyright $\odot 2012$ the authors $\quad 0270-6474 / 12 / 3212921-07 \$ 15.00 / 0$ frame of reference. Such areas would preactivate the brainstem HBR circuits when the stimulated hand is located within the peripersonal space of the face. Importantly, this increased excitability is specific for the brainstem interneurons mediating the HBR but not for those mediating the BR elicited by trigeminal stimulation or for the facial motoneurons innervating the orbicularis oculi (i.e., it is heterosegmentally specific) (Sambo et al., 2012). Here we addressed the following three questions.

First, is such cortical modulation specific for the HBR elicited by the stimulation of the hand located inside the peripersonal space of the face (i.e., is it homosegmentally specific?), or could it be observed also in the response elicited by the stimulation of the hand contralateral to the one near the face? We hypothesized that, because of the defensive function of the HBR, its top-down modulation by hand position (Sambo et al., 2012) is homosegmentally specific, that is, the cortex would preferably enhance the excitability of the circuits mediating the HBR elicited by the stimulation of the hand placed inside the peripersonal space of the face.

Second, is the strength of the cortical modulation of the excitability of the HBR circuits affected by the participants' expectations about whether or not the hand placed inside the facial peripersonal space could be stimulated? We hypothesized that the top-down modulation of the HBR circuits would be dependent on cognitive expectations, being more effective when participants have higher expectations that the hand inside the peripersonal space of the face will be stimulated.

Third, is the strength of the modulation influenced by the presence of objects protecting the face? We hypothesized that 
placing a thin wooden screen between the participants' face and their hand would reduce the extension of the peripersonal space of the face, thus preventing the HBR enhancement attributable to hand-face proximity.

\section{Materials and Methods}

Participants. Ten healthy volunteers (five women) aged between 25 and 37 years (mean $\pm \mathrm{SD}, 30.3 \pm 3.9$ years), all right-handed, participated in this study. They were recruited, depending on their availability, from a database of 15 "responders," i.e., participants showing a reproducible HBR. These responders were identified from 25 volunteers that were initially screened for the presence of HBR. Thus, the percentage of responders was 15 of $25=60 \%$, i.e., identical to what we observed in our previous paper ( 12 of $20=60 \%$; Sambo et al., 2012). Participants gave written informed consent before taking part in the study. The study was approved by the local ethics committee.

Stimulation and recording. Electrical stimuli were delivered to the median nerve at the wrist using a surface bipolar stimulator attached on the participants' wrist with a Velcro strap. Importantly, the stimulator provided constant-current pulses, thus making the trial-to-trial variability in the applied current negligible. The stimulus intensity was adjusted, in each participant, to elicit a clear HBR in three consecutive trials $(20-80$ $\mathrm{mA}$, mean of $42.5 \mathrm{~mA}$; ranging between 13 and 53 times the individual perceptual threshold, mean of 28) (Sambo et al., 2012). None of the participants reported pain during stimulation even at high stimulus intensities. The stimulus duration was $200 \mu \mathrm{s}$, and the interval between successive stimuli was $30 \mathrm{~s}$.

Electromyographic (EMG) activity was recorded from the orbicularis oculi muscle, bilaterally, using two pairs of surface electrodes with the active electrode over the mid lower eyelid and the reference electrode a few centimeters laterally to the outer canthus. Signals were amplified and digitized at a sampling rate of $8192 \mathrm{~Hz}$ (ISA 1004; Micromed) and stored for offline analysis.

Contribution of peripheral fiber populations to the HBR. Although none of the participants reported pain during stimulation, at the high stimulation intensities used to elicit the HBR we cannot exclude that populations of fibers other than $\mathrm{A} \beta$ (i.e., $\mathrm{A} \delta$ and $\mathrm{C}$ fibers) were recruited. However, the onset-offset latencies of the HBR ( $\sim 45-100$ ms; Sambo et al., 2012) rule out any contribution of $A \delta$ and $C$ afferents, because the conduction velocity of these fiber populations is not compatible with such HBR latencies (Plaghki et al., 2010). To illustrate this point and show the time profile of the contribution of different classes of somatosensory afferents to the HBR, we recorded, in six participants, the BR elicited by infrared laser stimuli applied to the hand dorsum (while the participant's arm was always kept far from the face). Such laser stimuli activate selectively nociceptive free nerve endings in the most superficial epidermal layers, without any concomitant activation of $\mathrm{A} \beta$ afferents (Bromm and Treede, 1984). The onset latency of the laser-evoked HBR was $\sim 130 \mathrm{~ms}$ (Fig. 1), i.e., later than the offset latency of the electrically evoked HBR. This evidence rules out that the electrically evoked HBR reflects the activation of $\mathrm{A} \delta$ - and/or $\mathrm{C}$-fiber primary somatosensory afferents.

Procedures. In all experiments, we recorded HBR responses while one of the two hands underwent the "far" and "near" postural manipulation used in our previous study (Sambo et al., 2012). In the far position, participants were sitting with their forearm resting on a pillow, at $\sim 120^{\circ}$ with respect to the arm and with the hand close to the ipsilateral knee (see Figs. 2,4 ); in the near position, participants were sitting with their arm resting on a table, the forearm at $\sim 75^{\circ}$ with respect to the arm, and the hand at a distance of $\sim 4 \mathrm{~cm}$ from the ipsilateral side of their face (see Figs. $2,4)$. The fingers, the hand, the wrist, or any other part of the upper limb were never touching the face or the head. Throughout each block, participants were instructed to keep their gaze on a fixation cross $(1.5 \times 1.5$ $\mathrm{cm}$ ) placed at $\sim 30 \mathrm{~cm}$ and $45^{\circ}$ below eye level. White noise was played throughout the experiment to mask any auditory cue possibly arising from the stimulation procedure.

In experiment 1, electrical stimuli were delivered, with equal probability, to either the hand undergoing the postural (i.e., "far" and "near")

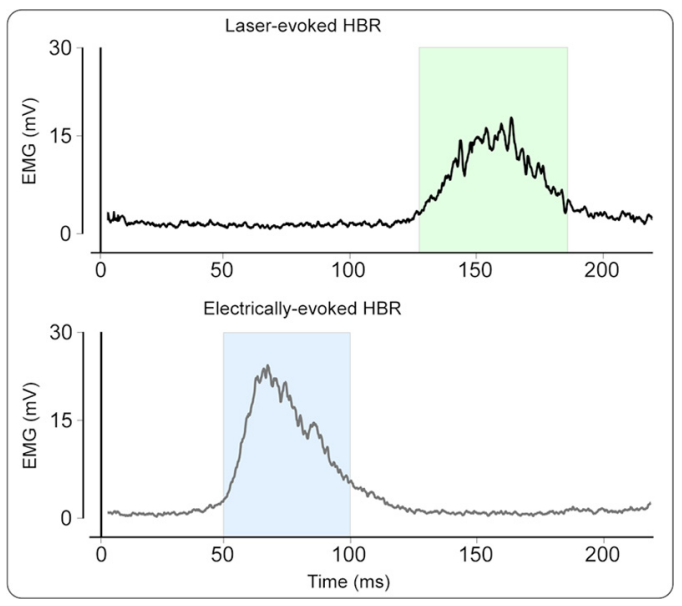

Figure 1. Group-averaged, rectified HBR waveforms elicited by laser stimuli (top) and electrical stimuli (bottom), while the hand was kept in the far position; $x$-axis, Time (milliseconds); $y$-axis, EMG (millivolts). Note that the onset latency of the laser-evoked HBR is $\sim 130 \mathrm{~ms}$, i.e., later than the offset latency of the electrically evoked HBR ( $\sim 100 \mathrm{~ms})$. Because laser stimuli activate selectively nociceptive afferents, without concomitant activation of $A \beta$ afferents, this evidence rules out that the electrically evoked HBR is contributed by the activation of $A \delta$ - and/or C-fiber primary somatosensory afferents.

manipulation ("moving hand") or the other hand ("non-moving hand") (see Fig. 2). The hand not undergoing the postural manipulation was always kept on the pillow in the far position. Participants were told at the beginning of the experiment that the electrical stimuli would be delivered to either hand with equal probability; however, they did not know in advance which hand would be stimulated on each trial. In two separate blocks, either the left or the right hand underwent the postural manipulation. A total of 64 electrical stimuli were delivered as follows: 32 stimuli to the moving hand ( 16 in the far condition and 16 in the near condition) and 32 stimuli to the non-moving hand, always kept in the far position throughout the block (16 while the moving hand was in the far condition and 16 while the moving hand was in the near condition). The stimuli were delivered in pseudorandom order, with no more than two consecutive stimuli delivered to the same hand.

In experiment 2, electrical stimuli were only delivered to the hand contralateral to the one undergoing the postural manipulation (nonmoving hand), while the moving hand was never stimulated (see Fig. 4). Participants were told at the beginning of the experiment that the electrical stimuli would be only delivered to the hand kept in the far position. In separate blocks, 16 stimuli were delivered to the left wrist and 16 to the right wrist, for a total of 32 stimuli. The order of blocks was balanced across participants. In each block, eight stimuli were delivered while the moving hand was kept in the far position and eight while this hand was in the near position, in alternating trials.

In experiment 3 , electrical stimuli were only delivered to the hand undergoing the far and near manipulation. We recorded HBR responses in two conditions: "screen" and "no screen." In the screen condition, a $400 \times 620 \times 3 \mathrm{~mm}$ (height $\times$ width $\times$ depth) wooden panel supported by two metal stands was positioned vertically on the table on which the arm was resting, at a distance of $\sim 4 \mathrm{~cm}$ from the participant's eye (see Fig. 5). The participant placed their arm under the panel, and the panel did not touch either the participant's arm or chest. The panel completely occluded the view of the forearm, the wrist, and the hand. In the noscreen condition, no screen was placed between the participant's eyes and their arm. The screen and no-screen conditions were performed in separate blocks: half of the participants performed the screen condition first and half started with the no-screen condition. For each of the screen and no-screen conditions, a total of 40 electrical stimuli were delivered to the median nerve, 20 stimuli to the left wrist and 20 stimuli to the right wrist, in separate blocks. In each block, 10 stimuli were delivered in the far and 10 in the near condition, in alternating trials. After completing the experiment, participants were asked to rate the level of "perceived threat of 


\section{Somatotopical specificity of HBR enhancement}

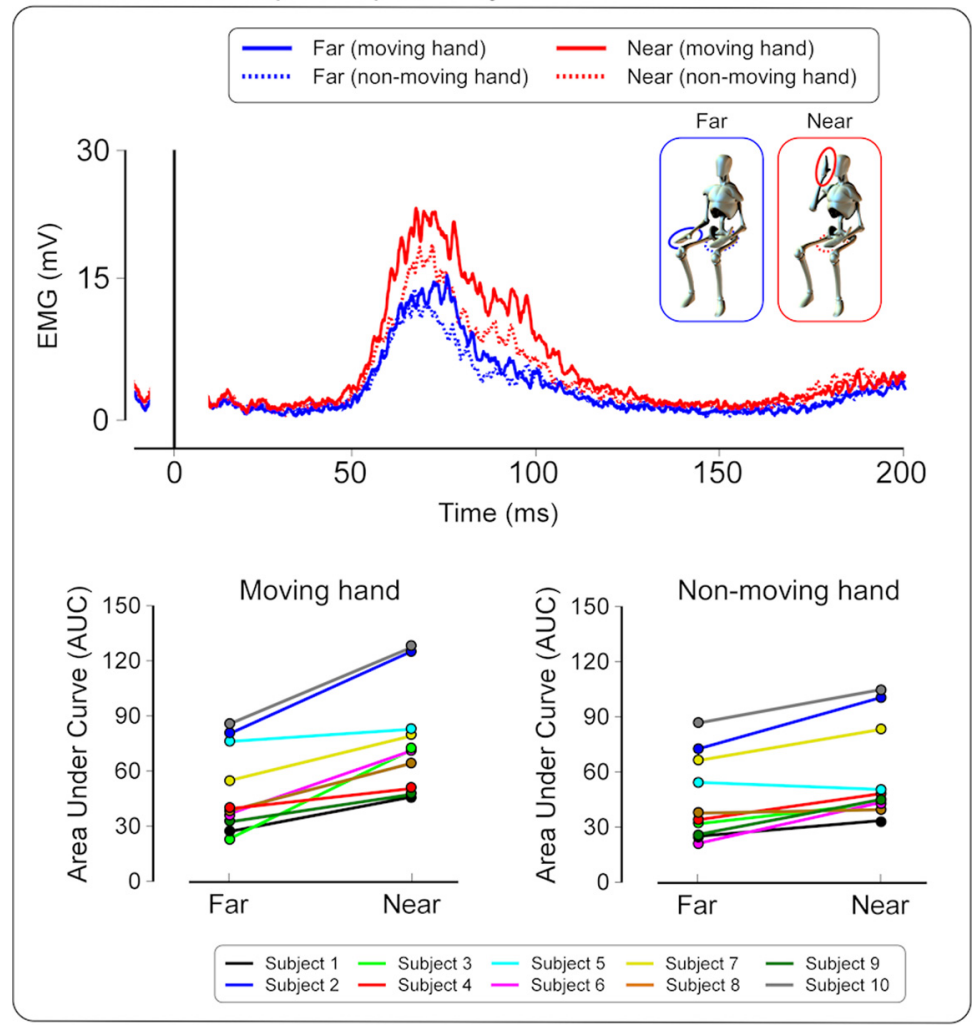

Figure 2. Experiment 1. Top, Group-averaged, rectified HBR waveforms elicited by the electrical stimulation of the hand undergoing the far and near manipulation (moving hand) and of the other hand (non-moving hand), always kept in the far position; $x$-axis, Time (milliseconds); $y$-axis, EMG activity (millivolts). Note that all waveforms are averaged according to the position (far vs near) of the moving hand. Thus, in the near condition (red), one hand was placed near the face (moving hand, solid line) and the other was kept far from the face (non-moving hand, dashed line), and stimuli were delivered to either hand with equal probability. In the far condition (blue), both hands were kept far from the face, and stimuli were delivered, with equal probability, to the moving hand (solid line) and to the non-moving hand (dashed line). Crucially, participants did not know in advance which of the two hands would be stimulated on each trial. Note that the HBR is significantly greater in the near than in the far condition, particularly in response to stimuli applied to the moving hand. Bottom, Single-subject HBR magnitudes (expressed as AUC arbitrary units) in the far and near conditions, for the moving hand and the non-moving hand.

stimulation" on an 11-point scale $(0=$ no threat to $10=$ maximum threat).

Data analyses and statistics. EMG signals in all experiments were analyzed using Letswave (http://nocions.webnode.com) (Mouraux and Iannetti, 2008). EMG signals from each participant were high-pass filtered $(55 \mathrm{~Hz})$ and full-wave rectified. In all experiments, data were averaged across ipsilateral and contralateral recording sides.

In experiment 1, HBR responses elicited by the stimulation of the moving hand and the non-moving hand were averaged separately, according to the position (far vs near) of the hand undergoing the postural manipulation (moving hand), resulting in four HBR average waveforms for each subject. In experiment 2, HBR responses elicited by the stimulation of the non-moving hand (the only hand that was stimulated in this experiment) were averaged according to the position of the moving hand. This resulted in two average waveforms for each subject. In experiment 3, HBR responses in the screen and no-screen conditions were averaged separately, according to the position (far vs near) of the hand undergoing the postural manipulation (the only hand that was stimulated in this experiment). This resulted in four average waveforms for each subject.

In each participant, we measured the area under the curve (AUC) of each HBR average waveform. In experiment 1, we performed a two-way, repeated-measures ANOVA, with "position of the moving hand" (two levels: far and near) and "stimulated hand" (two levels: moving hand and non-moving hand) as experimental factors. In experiment 2, we performed a paired $t$ test to compare the HBR waveforms in the far and near positions. In experiment 3, we performed a two-way, repeated-measures ANOVA, with "hand position" (two levels: far and near) and "screen" (two levels: screen and no screen) as experimental factors.

\section{Results \\ Somatotopical specificity of the HBR enhancement}

When the electrical stimuli were delivered to either the moving hand or the nonmoving hand with equal probability (experiment 1), the magnitude of the HBR was significantly larger when the stimulated hand was inside the peripersonal space of the face (main effect of position of the moving hand, $F_{(1,9)}=36.59, p<$ $0.001)$ and when the HBR was elicited by the stimulation of the hand undergoing the postural manipulation (moving hand) compared with the hand always kept in the far position (non-moving hand) (main effect of stimulated hand, $F_{(1,9)}=$ $11.42, p=0.008)$. Crucially, the interaction between the two factors was significant $\left(F_{(1,9)}=11.27, p=0.008\right)$, indicating that the increase of HBR magnitude in the near condition was significantly larger for the HBR elicited by the stimulation of the moving hand compared with the HBR elicited by the stimulation of the nonmoving hand $(+68.2 \pm 56.3$ vs $+38.1 \pm$ $33.1 \%$ increase; $t_{(9)}=3.36, p=0.008$; paired $t$ test) (Fig. 2).

\section{Effect of cognitive expectations on the HBR enhancement}

When participants were aware that the electrical stimuli would be only delivered to the non-moving hand (i.e., the probability of receiving stimuli on the moving hand was $0 \%$; experiment 2), the HBR magnitude was identical regardless of whether the contralateral, moving hand was placed inside or outside the peripersonal space of the face $\left(t_{(9)}=0.22, p=0.83\right.$; paired $t$ test $)$ (see Fig. 4).

Effect of the presence of the screen on the HBR enhancement When the wooden screen was placed between the participants' face and hand (experiment 3), the modulation of the HBR magnitude by hand position was entirely lost (see Fig. 5). There was a significant main effect of hand position $\left(F_{(1,9)}=33.50, p<\right.$ 0.001 ), indicating that overall the HBR was significantly larger when the stimulated hand was close to the face, and a main effect of screen $\left(F_{(1,9)}=8.16, p=0.019\right)$, indicating that overall the HBR was significantly larger when there was no screen. Crucially, there was a significant interaction between the two factors $\left(F_{(1,9)}=34.38, p<0.001\right)$, indicating that the HBR magnitude was increased in the near condition but only when there was no screen (no screen: $+101.3 \pm 71 \%$ increase; screen: $+4.1 \pm 20.2 \%$ increase).

Furthermore, participants rated the perceived threat of stimulation as significantly lower in the presence of the screen (3.2 \pm 
Two independent top-down modulations (of different strength) of the interneurons mediating the HBRs elicited by stimulation of the 'near' hand and of the non-moving hand
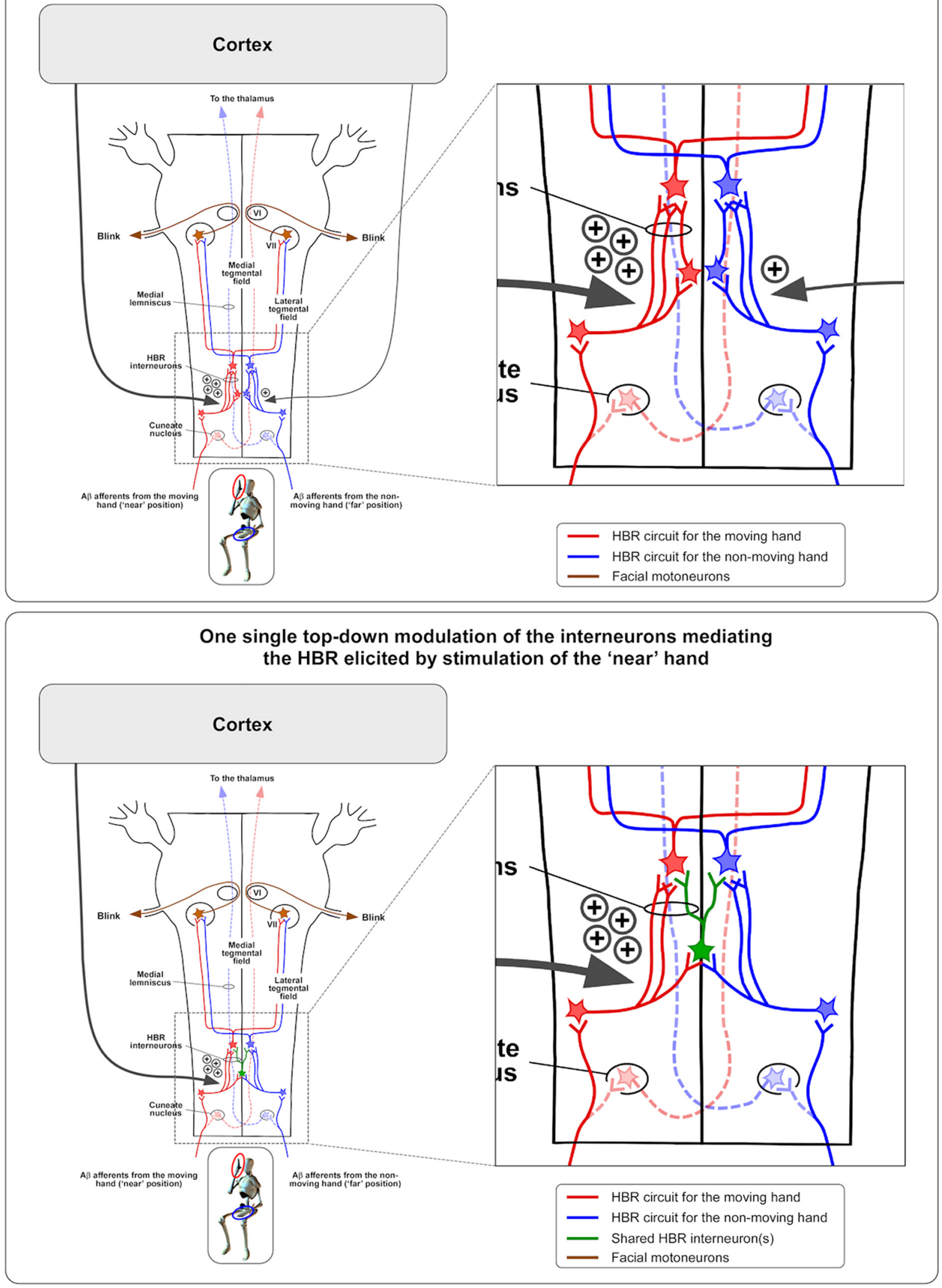

Figure 3. Two possible neural mechanisms responsible for the HBR enhancement observed when the moving hand (red) is placed inside the peripersonal space of the face (near position) and the non-moving hand (blue) is kept in the far position. Top, The interneurons of the brainstem circuits mediating the HBR elicited by the stimulation of the two hands might be (Figure legend continued.) 
Effect of cognitive expectations on HBR enhancement

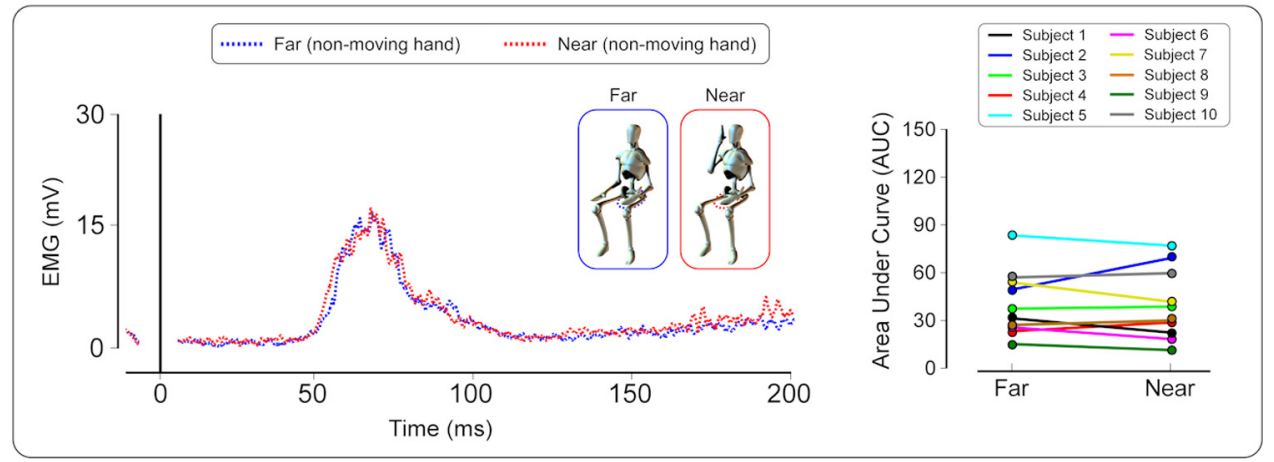

Figure 4. Experiment 2. Left, Group-averaged, rectified HBR waveforms elicited by the electrical stimulation of the hand always kept in the far position (non-moving hand); $x$-axis, time (milliseconds); $y$-axis, EMG activity (millivolts). Note that the waveforms are averaged according to the position (far vs near) of the moving hand, although this hand was never stimulated. (rucially, participants knew in advance that only the non-moving hand would be stimulated. Note that the HBR magnitude is remarkably similar in the near and the far condition. Right, Single-subject HBR magnitudes (expressed as AUC arbitrary units) in the far and near conditions, for the non-moving hand.

2.7) than when there was no screen $(5 \pm 2.6)\left(t_{(9)}=3.25, p=\right.$ 0.01 ; paired $t$ test).

\section{Discussion}

In this study, we characterized the somatotopical specificity of the HBR enhancement attributable to the spatial proximity between the stimulated hand and the face. We also investigated the effect of cognitive expectations and of the presence of objects protecting the face on such enhancement.

\section{Somatotopical specificity of the HBR enhancement by hand-face proximity}

The HBR enhancement observed when the stimulated hand is near the face (Sambo et al., 2012) is mediated by a tonic, topdown modulation exerted by cortical areas responsible for encoding the space surrounding the face on the brainstem interneurons mediating the HBR. Such modulation is specific for the interneurons mediating the HBR but not for those mediating the trigeminal BR (i.e., the modulation is heterosegmentally specific). The current results characterize further the somatotopical specificity of this modulation, because they show that such modulation is also partially specific when comparing inputs arising from homologous segments. Indeed, the cortex enhances preferentially the excitability of the circuits mediating the HBR elicited by the stimulation of the hand placed inside the peripersonal space of the face (resulting in an HBR enhancement of $+68.2 \%$ ) compared with the contralateral hand (resulting in an HBR enhancement of $+38.1 \%$ ) (Fig. 2). This finding highlights a re-

\section{$\leftarrow$}

(Figure legend continued.) entirely independent. This model implies two distinct cortical modulations, exerted with different strengths: one, stronger, on the circuit mediating the HBR elicited by the stimulation of the moving hand and the other, weaker, on the circuit mediating the HBR elicited by the stimulation of the non-moving hand. Bottom, The brainstem circuits mediating the HBR elicited by the stimulation of the two hands might share a subset of interneurons. This model allows a single top-down modulation directed uniquely to the brainstem interneurons mediating the HBR elicited by the stimulation of the moving hand. According to this model, the smaller enhancement of the HBR elicited by the stimulation of the nonmoving hand would be a byproduct of the neural architecture of the brainstem circuits subserving the two responses. Although it has not been investigated whether the circuits mediating the HBR elicited by the stimulation of the two hands are overlapping or separate, previous evidence that (1) the interneurons mediating the R2 response evoked by right and the left trigeminal stimuli are at least partially overlapping, and (2) the R2 and the HBR are functionally similar, suggests that the neural mechanisms represented in the bottom is more likely to account for the effects observed in experiment 1. markable ability of the neocortex to finely and selectively tune the excitability of different subcortical circuits mediating defensive responses. Indeed, considering the defensive nature of the BR, it makes functional sense that, when one hand is inside the peripersonal space of the face, only the circuits mediating the BR in response to somatosensory input from that hand become more excitable. In fact, a general increase of excitability of the BR elicited by stimuli applied to body districts other than those located close to the face would be evolutionary disadvantageous because it would trigger an unnecessary eye closure, interfering with effective responses in potentially dangerous situations.

What could be the neural mechanism responsible for the effects observed? Two alternative hypotheses may be put forward (Fig. 3). First, the cortical modulation might be directed to both the brainstem circuits mediating the HBR elicited by the stimulation of the hand entering the peripersonal space of the face and those mediating the HBR elicited by the stimulation of the contralateral hand, although the modulation of the latter would be less strong (Fig. 3, top). This hypothesis would assume that the interneurons of the brainstem circuits mediating the HBR elicited by the stimulation of the two hands are entirely independent, and it would imply two distinct modulations, exerted with different strengths. Alternatively, the cortical modulation might be directed uniquely to the brainstem interneurons mediating the HBR elicited by the stimulation of the hand placed inside the peripersonal space of the face, whereas the smaller enhancement of the HBR elicited by the stimulation of the contralateral hand would be a byproduct resulting from the neural architecture of the brainstem circuits subserving the two responses (Fig. 3, bottom). This second hypothesis would assume that the brainstem circuits mediating the HBR elicited by the stimulation of the two hands share a subset of their interneurons.

Although it has not been investigated whether the circuits mediating the HBR elicited by the stimulation of the two hands are overlapping or separate, there is evidence that the interneurons mediating the $\mathrm{R} 2$ response evoked by stimuli delivered to the right and the left trigeminal nerve are partly shared. Indeed, the $\mathrm{R} 2$ component of the BR elicited by the stimulation of the trigeminal nerve on one side is inhibited by a preceding stimulus delivered on the opposite side (Kimura and Harada, 1976). However, because in such condition the early R1 component of the BR is, instead, facilitated (Kimura and Harada, 1976), the R2 suppression must reflect an inhibition of the medullary interneurons and not of the facial motoneurons. Thus, considering the similarity 


\section{HBR with screen placed between the face and the hand}

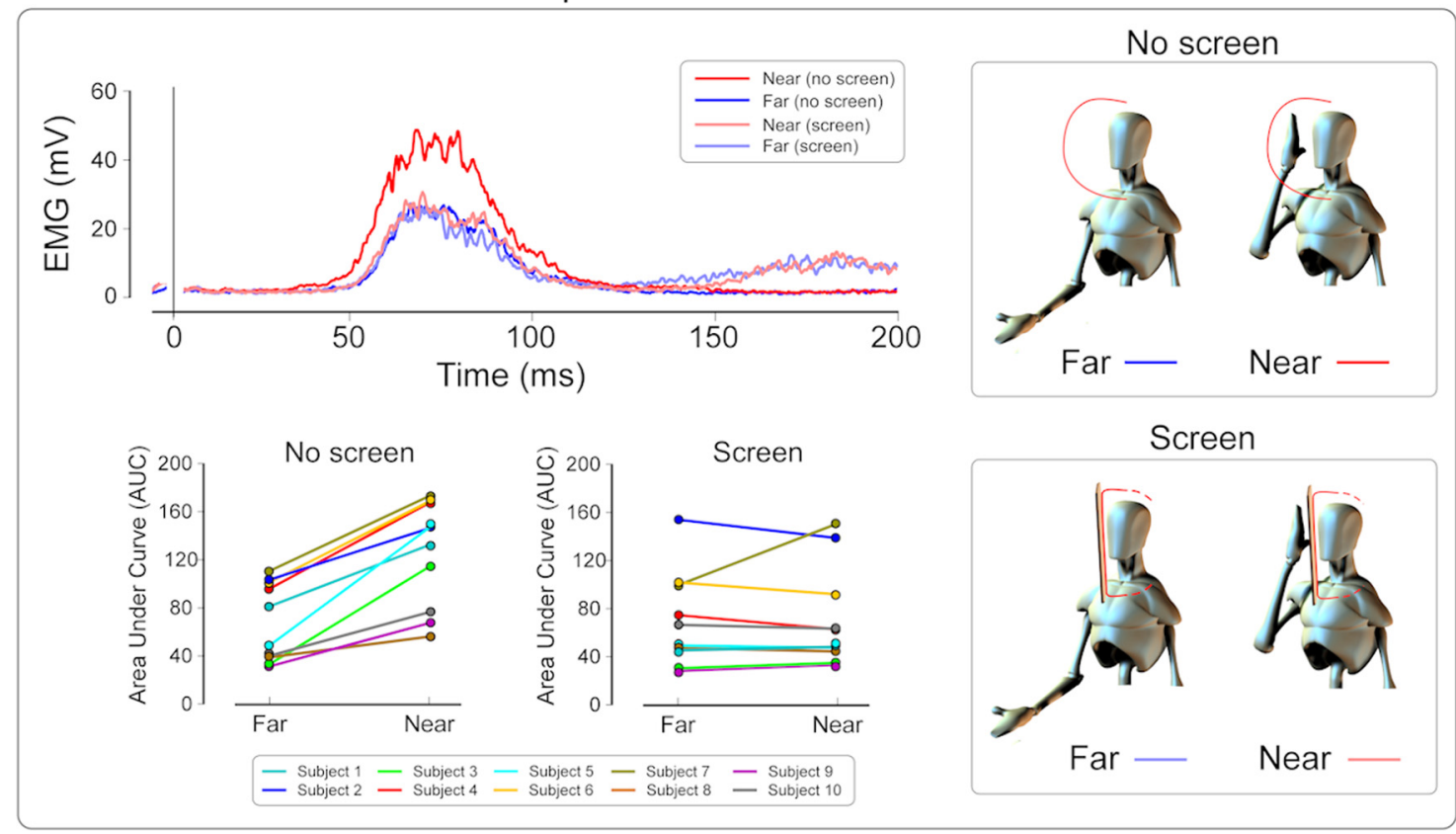

Figure 5. Experiment 3. Top, Group-averaged HBR waveforms in the far and near conditions when a thin wooden screen was placed close to the participants' face, thus separating it from their hand (screen condition) or there was no screen (no-screen condition); $x$-axis, Time (milliseconds); $y$-axis, EMG activity (millivolts). Note that the HBR enhancement by hand-face proximity is suppressed in the screen condition. This observation suggests that the presence of the screen reduces the boundaries of the peripersonal space of the face, resulting in the hand no longer entering the peripersonal space, even in the near condition. Bottom, Single-subject HBR magnitudes (expressed as AUC arbitrary units) in the far and near conditions for the screen and the no-screen conditions.

between the R2 component of the trigeminal BR and the HBR (Miwa et al., 1995; Valls-Solé et al., 1997; León et al., 2011) and the evidence that the circuits mediating the HBR and the trigeminal BR may interact at premotor level (Miwa et al., 1998), the results by Kimura and Harada (1976) provide support to the second of the hypotheses above, i.e., that the cortical modulation triggered by the hand-face proximity is homosegmentally specific, and that the smaller enhancement of the HBR elicited by the stimulation of the hand contralateral to that placed near the face (Fig. 3) would be the byproduct of the partial overlap between the interneurons mediating the HBR elicited by the stimulation of the left and right hand.

\section{Effect of cognitive expectations on HBR enhancement by hand-face proximity}

When participants knew that the electrical stimuli would never be applied to the hand undergoing the postural manipulation (experiment 2), the magnitude of the HBR elicited by the stimulation of the hand always kept in the far position was not modulated by the position of the hand changing its proximity to the face (Fig. 4). This finding indicates that the modulation reported by Sambo et al. (2012) and replicated here (experiment 1 ) is crucially dependent on expectations, i.e., it is only triggered when participants are aware that stimuli will be (Sambo et al., 2012) or could be (experiment 1) delivered to the hand entering the peripersonal space of the face. Furthermore, when participants do not have an a priori knowledge about which hand would receive the electrical stimuli (i.e., when the hand always kept far from the face and the hand changing its proximity to the face were stimulated with equal probability; experiment 1), the enhancement of the HBR elicited by the stimulation of the hand entering the peripersonal space of the face was smaller than that obtained previously
(Sambo et al., 2012) ( +68.2 vs $+99.3 \%$, on average). Together, these findings indicate that the cortex is able to finely adjust the level of excitability of the brainstem circuits as a function of the probability of stimulus occurrence.

We suggested previously that the perceived threat of stimuli applied to the hand increases when the hand is inside the peripersonal space surrounding the face, which results in the enhancement of the HBR (Sambo et al., 2012). Thus, we identified a defensive peripersonal space in humans, functionally distinct from the peripersonal space defined by multisensory integration and action control (Macaluso and Maravita, 2010). This defensive peripersonal space would represent a "safety margin" to protect the individual from external danger (Cooke and Graziano, 2003; Graziano and Cooke, 2006): whenever a potentially threatening stimulus approaches or enters this margin, the individual would detect and react to such stimuli more effectively. Here we suggest that perceived threat is modulated not only by the position of the stimulus with respect to the peripersonal space but also by the expectation of stimulus occurrence, with perceived threat being increased at high probabilities of stimulus occurrence. Accordingly, the HBR enhancement was maximal when the probability that the hand close to the face was going to be stimulated was set at $100 \%$ (Sambo et al., 2012) and reduced when the probability was set at 50\% (experiment 1 ). Moreover, the HBR was not modulated by the hand-face proximity when the probability of the hand close to the face being stimulated was set to $0 \%$ (experiment 2).

\section{Effect of the screen on the HBR enhancement by hand-face proximity}

When a thin wooden screen was placed close to the participants' face, thus separating it from their hand (experiment 3), the HBR enhancement by hand-face proximity vanished (Fig. 5). This re- 
sult suggests that the presence of the screen changes the boundaries of the peripersonal space of the face, resulting in the hand no longer entering the peripersonal space, even in the near condition (Fig. 5). Thus, the lack of modulation of the HBR by hand position may occur because, in both the far and near conditions, the hand is outside the facial peripersonal space, reduced in extension because of the screen. The extension of the peripersonal space defined by multisensory effects is dramatically modulated by the presence of objects. For example, it is increased by tools held with the hands (Holmes et al., 2004; Farne et al., 2007) and by the observation of body parts reflected in a mirror (Maravita et al., 2002; Sambo and Forster, 2011). Such modulation of the peripersonal space boundaries has been suggested to be mediated by rapid and short-lived neural plasticity in cortical areas, such as the posterior parietal cortex and the prefrontal cortex (Inoue et al., 2001; Iriki et al., 2001; Magosso et al., 2010; Cuppini et al., 2011). Although the defensive peripersonal space we characterized is probably different from the peripersonal space related to multisensory integration (Sambo et al., 2012), our result suggests that similar effects of short-term plasticity might take place in such frontoparietal areas and thus account for the lack of topdown modulation in the presence of the screen.

Importantly, the lack of modulation of the HBR in the presence of the screen cannot be accounted for by the effect of not viewing the hand. Indeed, the near/far modulation of the HBR is entirely unaffected when the eyes are closed, indicating that proprioceptive information is sufficient to determine an HBR enhancement of magnitude similar to the one observed when the eyes are open (Sambo et al., 2012).

\section{References}

Alvarez-Blanco S, Leon L, Valls-Solé J (2009) The startle reaction to somatosensory inputs: different response pattern to stimuli of upper and lower limbs. Exp Brain Res 195:285-292.

Bromm B, Treede RD (1984) Nerve fibre discharges, cerebral potentials and sensations induced by $\mathrm{CO} 2$ laser stimulation. Human neurobiology 3:33-40.

Cooke DF, Graziano MS (2003) Defensive movements evoked by air puff in monkeys. J Neurophysiol 90:3317-3329.

Cuppini C, Magosso E, Ursino M (2011) Organization, maturation, and plasticity of multisensory integration: insights from computational modeling studies. Frontiers in psychology 2:77.

Farne A, Serino A, Ladavas E (2007) Dynamic size-change of peri-hand space following tool-use: determinants and spatial characteristics re- vealed through cross-modal extinction. Cortex; a journal devoted to the study of the nervous system and behavior 43:436-443.

Graziano MS, Cooke DF (2006) Parieto-frontal interactions, personal space, and defensive behavior. Neuropsychologia 44:845-859.

Holmes NP, Calvert GA, Spence C (2004) Extending or projecting peripersonal space with tools? Multisensory interactions highlight only the distal and proximal ends of tools. Neurosci Lett 372:62-67.

Inoue K, Kawashima R, Sugiura M, Ogawa A, Schormann T, Zilles K, Fukuda $\mathrm{H}$ (2001) Activation in the ipsilateral posterior parietal cortex during tool use: a PET study. Neuroimage 14:1469-1475.

Iriki A, Tanaka M, Obayashi S, Iwamura Y (2001) Self-images in the video monitor coded by monkey intraparietal neurons. Neuroscience research 40:163-173.

Kimura J, Harada O (1976) Recovery curves of the blink reflex during wakefulness and sleep. J Neurol 213:189-198.

León L, Casanova-Molla J, Lauria G, Valls-Solé J (2011) The somatosensory blink reflex in upper and lower brainstem lesions. Muscle Nerve 43:196-202.

Macaluso E, Maravita A (2010) The representation of space near the body through touch and vision. Neuropsychologia 48:782-795.

Magosso E, Ursino M, di Pellegrino G, Làdavas E, Serino A (2010) Neural bases of peri-hand space plasticity through tool-use: insights from a combined computational-experimental approach. Neuropsychologia 48:812-830.

Maravita A, Spence C, Sergent C, Driver J (2002) Seeing your own touched hands in a mirror modulates cross-modal interactions. Psychol Sci 13:350-355.

Miwa H, Imamura N, Kogahara K, Ohori T, Mizuno Y (1995) Somatosensory evoked blink response: findings in patients with Miller Fisher syndrome and in normal subjects. J Neurol Neurosurg Psychiatry 58:95-99.

Miwa H, Yamaji Y, Abe H, Mizuno Y (1996) Evaluation of the somatosensory evoked blink response in patients with neurological disorders. J Neurol Neurosurg Psychiatry 60:539-543.

Miwa H, Nohara C, Hotta M, Shimo Y, Amemiya K (1998) Somatosensoryevoked blink response: investigation of the physiological mechanisms. Brain 121:281-291.

Mouraux A, Iannetti GD (2008) Across-trial averaging of event-related EEG responses and beyond. Magnetic resonance imaging 26:1041-1054.

Plaghki L, Decruynaere C, Van Dooren P, Le Bars D (2010) The fine tuning of pain thresholds: a sophisticated double alarm system. PLoS One 5:e10269.

Sambo CF, Forster B (2011) When far is near: ERP correlates of crossmodal spatial interactions between tactile and mirror-reflected visual stimuli. Neurosci Lett 500:10-15.

Sambo CF, Liang M, Cruccu G, Iannetti GD (2012) Defensive peripersonal space: the blink reflex evoked by hand stimulation is increased when the hand is near the face. J Neurophysiol 107:880-889.

Valls-Solé J, Valldeoriola F, Tolosa E, Marti MJ (1997) Distinctive abnormalities of facial reflexes in patients with progressive supranuclear palsy. Brain 120:1877-1883. 